\title{
Photovoltaic nanopillar radial junction diode architecture enhanced by integrating semiconductor quantum dot nanocrystals as light harvesters
}

Burak Güzeltürk, Evren Mutlugün, Xiaodong Wang, Kin Leong Pey, and Hilmi Volkan Demir

Citation: Appl. Phys. Lett. 97, 093111 (2010);

View online: https://doi.org/10.1063/1.3485294

View Table of Contents: http://aip.scitation.org/toc/apl/97/9

Published by the American Institute of Physics

\section{Articles you may be interested in}

A transferable force field for CdS-CdSe-PbS-PbSe solid systems

The Journal of Chemical Physics 141, 244503 (2014); 10.1063/1.4904545

Acoustic phonon strain induced mixing of the fine structure levels in colloidal CdSe quantum dots observed by a polarization grating technique

The Journal of Chemical Physics 132, 104506 (2010); 10.1063/1.3350871

Photoluminescence spectroscopy and lifetime measurements from self-assembled semiconductor-metal nanoparticle hybrid arrays

Applied Physics Letters 97, 083307 (2010); 10.1063/1.3483162

Si nanopillar array optimization on Si thin films for solar energy harvesting

Applied Physics Letters 95, 033102 (2009); 10.1063/1.3186046

Vapor-phase nucleation of individual CdSe nanostructures from shape-engineered nanocrystal seeds Applied Physics Letters 92, 023106 (2008); 10.1063/1.2825425

Efficient nonradiative energy transfer from InGaN/GaN nanopillars to CdSe/ZnS core/shell nanocrystals Applied Physics Letters 98, 163108 (2011); 10.1063/1.3562035

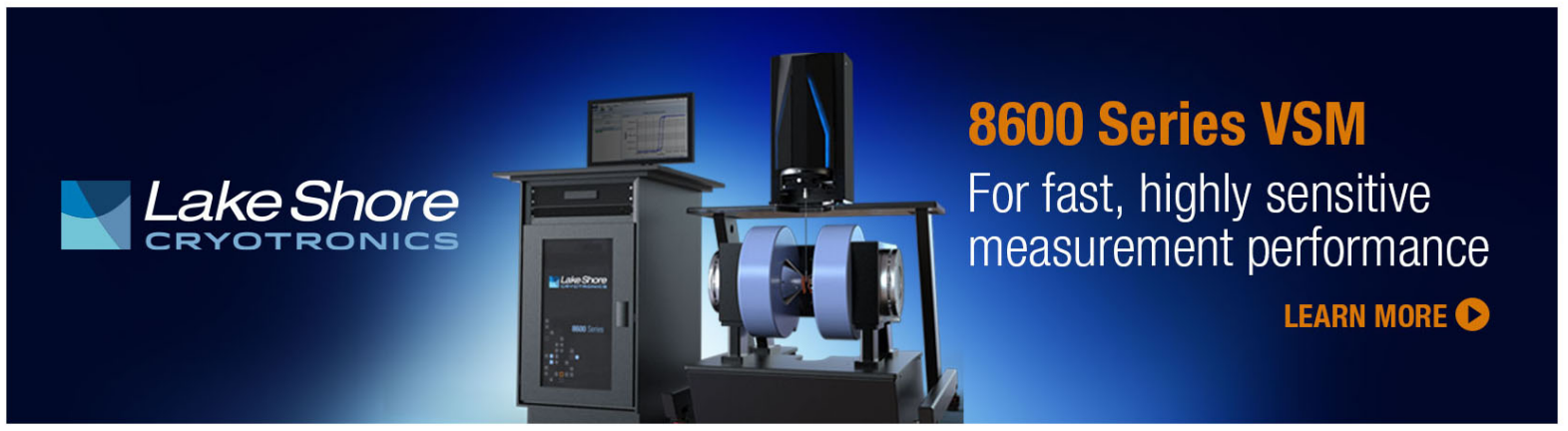




\title{
Photovoltaic nanopillar radial junction diode architecture enhanced by integrating semiconductor quantum dot nanocrystals as light harvesters
}

\author{
Burak Güzeltürk, ${ }^{1}$ Evren Mutlugün, ${ }^{1}$ Xiaodong Wang, ${ }^{3}$ Kin Leong Pey, ${ }^{2,3}$ and \\ Hilmi Volkan Demir ${ }^{1,2, a)}$ \\ ${ }^{1}$ Department of Physics, Department of Electrical and Electronics Engineering, and UNAM-National \\ Nanotechnology Research Center, Institute of Materials Science and Nanotechnology, Bilkent University, \\ Ankara TR-06800, Turkey \\ ${ }^{2}$ School of Electrical and Electronic Engineering Division of Microelectronics, School of Physical and \\ Mathematical Sciences, Division of Physics and Applied Physics, Nanyang Technological University, \\ Nanyang Avenue, Singapore 639798 \\ ${ }^{3}$ Advanced Materials for Micro- and Nano-systems Programme, Singapore-MIT Alliance, Singapore 117576
}

(Received 22 June 2010; accepted 10 August 2010; published online 3 September 2010)

\begin{abstract}
We propose and demonstrate colloidal quantum dot hybridized, radial $p-n$ junction based, nanopillar solar cells with photovoltaic performance enhanced by intimately integrating nanocrystals to serve as light harvesting agents around the light trapping pillars. By furnishing Si based nanopillar photovoltaic diodes with CdSe quantum dots, we experimentally showed up to sixfold enhancement in UV responsivity and $\sim 13 \%$ enhancement in overall solar conversion efficiency. The maximum responsivity enhancement achieved by incorporation of nanocrystals in the nanopillar architecture is found to be spectrally more than four times larger than the responsivity enhancement obtained using planar architecture of the same device. (C) 2010 American Institute of Physics.
\end{abstract}

[doi:10.1063/1.3485294]

The Sun's enormous potential as a solar conversion energy source has recently led to important research efforts in photovoltaics. ${ }^{1}$ Although the photovoltaic market is currently being dominated by conventional, silicon based solar cells (also known as the first generation solar cells), ${ }^{2}$ alternative device structures that can potentially enable efficient solar conversion at reduced costs are heavily being investigated. Today one dimensional nanostructures such as nanowires and nanorods are gradually coming into prominence in photovoltaics research. ${ }^{3-6} \mathrm{Si}$ nanopillar solar cells are promising candidates as they can harness the advantages of onedimensional confined structures including enhanced photocurrent (owing to increased junction area with a large surface-to-volume ratio) and improved optical properties (including light trapping, and higher absorption and lower reflectance with respect to their thin film counterparts). ${ }^{7-11}$ However, the-state-of-art efficiencies of these nanostructured solar cells are typically lower than their planar versions due to the problems encountered in nanofabrication processes and difficulties related to their poor surface passivation. ${ }^{4} \mathrm{Al}-$ though such nanofabrication methods can be matured in the future, it is technically challenging to overcome the intrinsic limitations. Such one important limitation is that $\mathrm{Si}$ based solar cells suffer from poor responsivity at short wavelengths, particularly in UV and blue. ${ }^{12}$ However, this unused spectral range of the solar irradiation constitutes $\sim 10 \%$ of sun light. ${ }^{13}$ To provide a solution to this low responsivity and light harvesting problem at short wavelengths, we propose and demonstrate utilization of colloidal quantum dots as a wavelength up-converting layer hybridized on radial $p$ - $n$ junction nanopillar solar cells made of $\mathrm{Si}$ to improve their

\footnotetext{
${ }^{\text {a) }}$ Authors to whom correspondence should be addressed. Electronic addresses: volkan@bilkent.edu.tr and hvdemir@ntu.edu.sg.
}

UV performance and enhance their solar conversion efficiency.

There have been previous reports focusing on the use of luminescent wavelength up-converters thus far, including quantum dots to harvest light spectrally better to improve the performance of $\mathrm{Si}$ based photodetectors ${ }^{14}$ and photovoltaic devices. ${ }^{15-18}$ However, to date, the class of new solar cell architectures based on nanopillars have not been investigated for the hybridization of quantum dots as optical wavelength up-converters to enhance solar conversion activity. With incorporation of such quantum dots, structural advantages of nanopillars including light trapping can also be benefited to obtain higher levels of enhancement compared to planar solar cell counterparts.

In this work, we investigate the integration of $\mathrm{CdSe}$ nanocrystals intimately on radial $p-n$ junction based Si nanopillar solar cells, with these nanocrystals strongly absorbing incident UV and blue light and emitting at a long wavelength, which was chosen to match the higher spectral response of the silicon solar cell. Through hybridization of these colloidal quantum dots on Si nanopillar solar cells, we have demonstrated approximately $13 \%$ enhancement of overall solar conversion efficiency measured under AM1.5G (air mass 1.5 global) conditions. We have also shown spectral enhancement up to sixfolds in responsivity of the nanopillar photovoltaic devices measured under monochromatic UV light illumination. Comparing against the planar architecture of the same silicon solar cells with the same amount of hybridized quantum dots, we have shown that a maximum enhancement factor of more than four times can be achieved using the nanopillar architecture, because of its superior geometric and structural characteristics.

In our experiments, scanning electron microscopy (SEM) images are taken by FEI Quanta 200 FEG, and transmission electron microscopy (TEM) images, by FEI Tecnai G2 F30. Absorbance and emission spectra are recorded using 
(a)

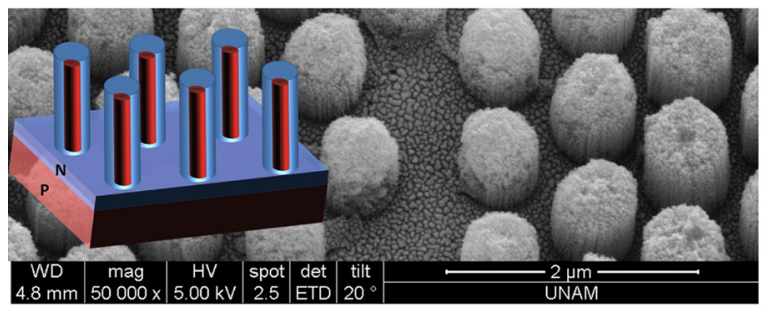

(b)

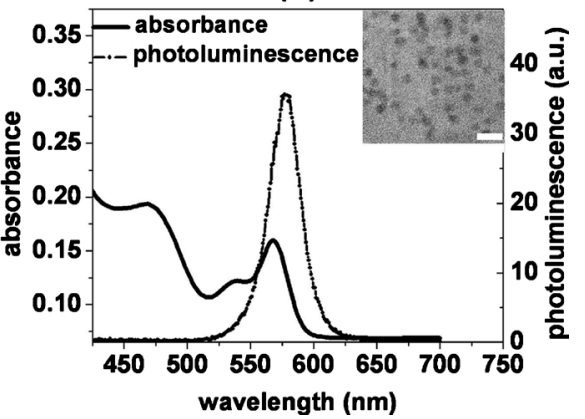

FIG. 1. (Color online) (a) SEM image of nanopillars in the solar cell, together with a schematic of radial $p$ - $n$ junction in nanopillar structure (inset), (b) emission and absorption spectra of colloidal CdSe nanocrystals in solution, along with a TEM image (inset). (The scale bar is $10 \mathrm{~nm}$ ).

Cary UV-Vis spectrophotometer and Cary 100 Fluorometer, respectively. A dynamic light scattering (DLS) system from Malvern Zeta Sizer is used for the quantum dot size characterization, and a Newport Series $150 \mathrm{~W}$ Solar Simulator is employed for photovoltaic performance characterizations. Here, for solar cell testing, the standard sunlight spectrum is simulated at an intensity level of $100 \mathrm{~mW} / \mathrm{cm}^{2}$ incident on the Earth surface by using an AM1.5G filter (air mass 1.5 global filter) in the solar simulator to include both diffusive and direct sunlight radiation Subsequently, external quantum efficiency (EQE) and responsivity of these devices are investigated using a wide spectrum Xe light source, a monochromator, a chopper, an optical powermeter to observe the spectral enhancement effects of the up-converter nanocrystal layers.

Figure 1(a) shows a SEM image of Si nanopillar solar cell. The pillar diameter is around $670 \mathrm{~nm}$ with a pillar height over $1 \mu \mathrm{m}$. These are radial $p$ - $n$ junction coaxial solar cells fabricated by diffusion process based on metal assisted chemical top-down etching method ${ }^{19,20}$ (see Ref. 24 for further description of the device processing). Figure 1(b) shows the absorption and photoluminescence spectra of colloidal CdSe nanocrystals in solution, along with a TEM image. These quantum dots are synthesized in organic nonpolar solvent using hot-injection technique ${ }^{21,22}$ (see Ref. 24 for the details of quantum dot synthesis). The first exciton peak of CdSe nanocrystals corresponds to $568 \mathrm{~nm}$ with a peak emission wavelength of $579 \mathrm{~nm}$. The photoluminescence quantum yield of nanocrystals is measured as 35\% (using rhodamine $6 \mathrm{G}$ as a reference dye). The size of the core $\mathrm{CdSe}$ quantum dots is calculated to be $3.46 \mathrm{~nm}$ (Ref. 23) and ex-

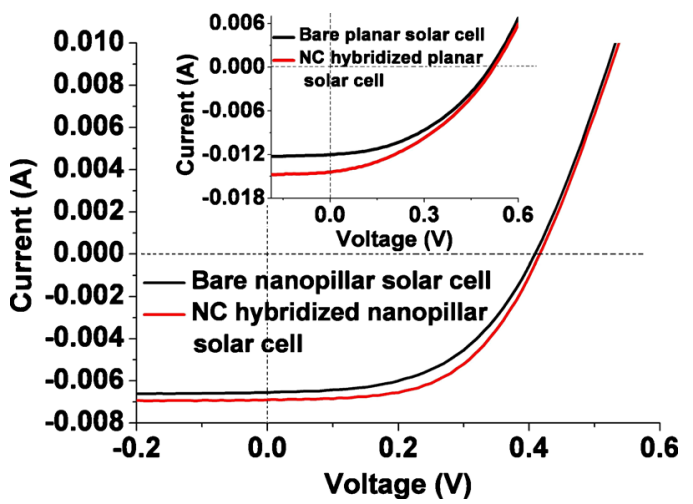

FIG. 2. (Color online) Current-voltage $(I-V)$ characteristics of nanopillar solar cells and that of planar solar cells (inset), both under AM1.5G conditions, before and after incorporation of wavelength up-converting nanocrystals. perimentally observed to be $7.65 \mathrm{~nm}$ using DLS; here the diameter is observed to be larger possibly due to the ligands attached on the surface of the quantum dots.

To employ the quantum dots as optical wavelength upconverting layer, we have coated CdSe nanocrystals over solar cells both in nanopillar and planar architectures using drop-casting method. The hybridized amount of nanocrystals is identical for both architectures (which is empirically found to be $81.74 \mu \mathrm{g} / \mathrm{cm}^{2}$ in the optimal case). The currentvoltage $(I-V)$ characteristics of solar cells are measured using solar simulator under AM1.5G conditions to study the evolution of solar cell parameters before and after nanocrystal integration. Figure 2 shows $I-V$ curves of both planar and nanopillar architectures measured under AM1.5G conditions. Hybridization of CdSe nanocrystals on both of the solar cell structures increased the short circuit current $\left(I_{\mathrm{sc}}\right)$ as expected, as a result of the enhancement in responsivity of the respective hybrid devices. Open circuit voltage $\left(V_{\text {oc }}\right)$ remained approximately the same before and after the nanocrystal incorporation. The solar conversion efficiency is increased by $13.37 \%$ and $12.42 \%$ in nanopillar solar cell and thin film planar solar cell architectures, respectively. This enhancement stems from the enhanced $\mathrm{EQE}$ of the hybrid devices. Compared to the planar solar cells, the enhancement achieved with nanopillar solar cells is larger since the nanopillar solar cell provides a suitable geometry for light trapping. In the nanopillar architecture light emitted by the nanocrystals is mostly trapped, which facilitates stronger absorption of these emitted photons in $\mathrm{Si}$, whereas at least half of the emitted photons from nanocrystals cannot be harvested in the planar case. Table I summarizes the evolution of solar cell parameters for both nanopillar and planar architectures.

Furthermore, to gain more physical insight on the origin of the enhancement, we have used monochromatic light, and measured the EQE of the devices. When nanocrystals are hybridized on the solar cell platform, it is shown that higher energy photons corresponding to the ultraviolet and blue

TABLE I. Solar cell parameters of nanopillar and planar silicon solar cell architectures before and after CdSe nanocrystal integration.

\begin{tabular}{lrrrr}
\hline \hline & $\begin{array}{c}I_{\mathrm{SC}} \\
(\mathrm{mA})\end{array}$ & $\begin{array}{l}V_{\mathrm{OC}} \\
(\mathrm{V})\end{array}$ & $\begin{array}{c}\text { Fill factor } \\
(\%)\end{array}$ & $\begin{array}{c}\text { Efficiency } \\
(\%)\end{array}$ \\
\hline Nanopillar solar cell without nanocrystals & 6.56 & 0.42 & 50.4 & 1.72 \\
Nanopillar solar cell with nanocrystals & 6.90 & 0.42 & 54.5 & 1.95 \\
Planar solar cell without nanocrystals & 12.01 & 0.52 & 41.95 & 3.22 \\
Planar solar cell with nanocrystals & 14.43 & 0.53 & 38.3 & 3.62 \\
\hline \hline
\end{tabular}




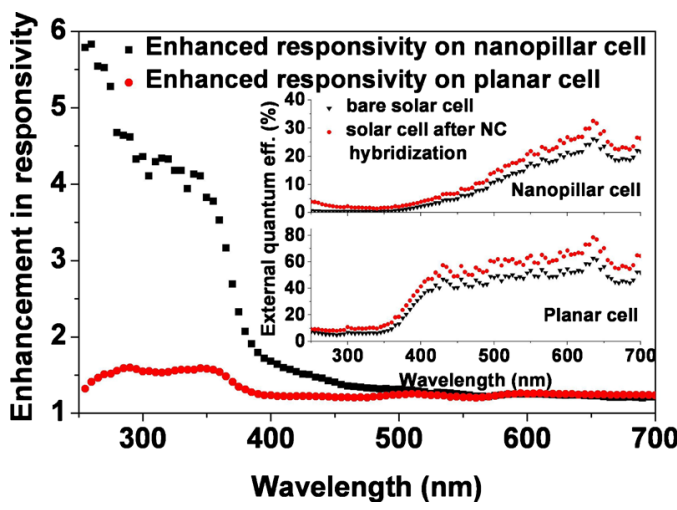

FIG. 3. (Color online) Optical responsivity enhancement of Si based planar and nanopillar solar cell architectures. The inset shows the EQE of the nanopillar and planar solar cells, both examined before and after quantum dot integration.

range of the solar spectrum are utilized more efficiently (see Fig. 3). EQE spectra of nanopillar and planar solar cell architectures are also depicted before and after hybridization of nanocrystal layer in Fig. 3 (inset). Here we show that the nanocrystals provide up to sixfold EQE enhancement in the UV portion of solar light on the nanopillar solar cell platform (see Fig. 3). The maximum EQE enhancement achieved using nanopillar architecture is found to be more than 4 times larger compared to the planar structure. This stems from the superior light trapping of the nanopillar structure revealed by the use of nanocrystals.

Although CdSe nanocrystals have no emission at longer wavelengths, there is still an enhancement about $20 \%$ in EQE of both nanopillar and planar devices at these wavelengths. It is found that introduced nanocrystal thin film over silicon serves as a graded index layer, which also increases the coupling of light into solar cell even at long wavelengths where nanocrystals do not emit, as confirmed by reflectivity measurements. We observe that the reflection of Si nanopillar solar cells drops off with the integration of quantum dots in the range of $9 \%-14 \%$ (see Ref. 24).

Due to immature fabrication methods, EQE of nanopillar solar cells is lower than that of thin film devices (see Fig. 3). As a result, a planar solar cell typically has higher solar conversion efficiency than a nanopillar solar cell. The problem of large surface states in nanopillar devices undesirably leads to higher recombination rates for photogenerated electron-hole pairs, which in turn results in lower EQE. When a wavelength up-converting nanocrystal layer is incorporated, although the resulting EQE enhancement in nanopillar structure is much larger than that in planar device because of better light trapping of nanopillars, this enhancement is not fully reflected in the overall solar conversion efficiency enhancement. This is due to fact that smaller enhancement in EQE of planar device contributes more additional photocurrent than the additional amount contributed in the case of nanopillar device, while the starting $\mathrm{EQE}$ is much larger in the planar device. Ideally, with nanopillar solar cells having similar EQE as the planar solar cells, much larger enhancements in solar conversion efficiency can be obtained in nanopillar architecture.

In summary, we studied and showed enhanced photovoltaic device performance by hybridizing CdSe nanocrystals on radial $p-n$ junction based silicon nanopillar solar cells. In such hybrid architecture, these nanocrystals are utilized as efficient wavelength up-converters to harvest incident photons that are otherwise poorly utilized at short wavelengths and convert them to photons favorably used at long wavelengths by silicon solar cells. We demonstrated approximately $13 \%$ enhancement of solar conversion efficiency under AM1.5G condition and up to sixfold enhancement in responsivity in UV spectrum for the nanopillar solar cells. Additionally, we achieved a maximum responsivity enhancement of more than fourfolds in UV using the nanopillar solar cells compared to the planar cell case. This is enabled by the superior light trapping properties of nanopillar architecture. Radial junction nanopillar solar cells hold promise for possible future photovoltaic applications with their performance enhanced via the hybridization of optical wavelength upconverting nanocrystal quantum dots around the pillars.

This work is supported by Grant No. NRF RF 2009-09, EU-FP7 Nanophotonics4Energy NoE, and TUBITAK Grant Nos. EEEAG 107E088, 109E002, 109E004, and 110E010. H.V.D. acknowledges support from ESF-EURYI and TUBAGEBIP, and E.M. and B.G. from TUBITAK-BIDEB. X.W. and K.L.P. acknowledge support from Singapore-MIT Alliance (SMA), Singapore. B.G. and E.M. contributed equally to this work.

${ }^{1}$ J. Zhu and Y. Cui, Nature Mater. 9, 183 (2010).

${ }^{2}$ A. Müller, M. Ghosha, R. Sonnenschein, and P. Woditsch, Mater. Sci. Eng., B 134, 257 (2006).

${ }^{3}$ T. Stelzner, M. Pietsch, G. Andra, F. Falk, E. Ose, and S. Christiansen, Nanotechnology 19, 295203 (2008).

${ }^{4}$ E. C. Garnett and P. D. Yang, J. Am. Chem. Soc. 130, 9224 (2008).

${ }^{5}$ L. Tsakalakos, J. Balch, J. Fronheiser, B. A. Korevaar, O. Sulima, and J. Rand, Appl. Phys. Lett. 91, 233117 (2007).

${ }^{6}$ B. Z. Tian, X. L. Zheng, T. J. Kempa, Y. Fang, N. F. Yu, G. H. Yu, J. L. Huang, and C. M. Lieber, Nature (London) 449, 885 (2007).

${ }^{7}$ K. Peng, Y. Xu, Y. Wu, Y. Yan, S.-T. Lee, and J. Zhu, Small 1, 1062 (2005)

${ }^{8}$ J. Li, H. Y. Yu, S. M. Wong, G. Zhang, X. Sun, P. G.-Q. Lo, and D.-L. Kwong, Appl. Phys. Lett. 95, 033102 (2009).

${ }^{9}$ J. Zhu, Z. Yu, G. F. Burkhard, C.-M. Hsu, S. T. Connor, Y. Xu, Q. Wang, M. McGehee, S. Fan, and Y. Cui, Nano Lett. 9, 279 (2009).

${ }^{10}$ M. D. Kelzenberg, S. W. Boettcher, J. A. Petykiewicz, D. B. T. Evans, M C. Putnam, E. L. Warren, J. M. Spurgeon, R. M. Briggs, N. S. Lewis, and H. A. Atwater, Nature Mater. 9, 239 (2010).

${ }^{11}$ J. Zhu, C.-M. Hsu, Z. Yu, S. Fan, and Y. Cui, Nano Lett. 10, 1979 (2010).

${ }^{12}$ S. M. Sze, Physics of Semiconductor Devices, 2nd ed. (Wiley, New York, 1981).

${ }^{13}$ ASTM G173-03 Reference Spectra, National Renewable Energy Laboratory (NREL) Reference Solar Spectral Irradiance.

${ }^{14}$ E. Mutlugün, I. M. Soganci, and H. V. Demir, Opt. Express 15, 1128 (2007).

${ }^{15}$ E. Mutlugün, I. M. Soganci, and H. V. Demir, Opt. Express 16, 3537 (2008).

${ }^{16}$ T. Trupke, M. A. Green, and P. Würfel, J. Appl. Phys. 92, 1668 (2002).

${ }^{17}$ W. G. J. H. M. van Sark, Appl. Phys. Lett. 87, 151117 (2005).

${ }^{18}$ B. S. Richards, Sol. Energy Mater. Sol. Cells 90, 2329 (2006).

${ }^{19}$ Z. Huang, H. Fang, and J. Zhu, Adv. Mater. 19, 744 (2007).

${ }^{20}$ K. Peng, M. Zhang, and A. Lu, Appl. Phys. Lett. 90, 163123 (2007).

${ }^{21}$ Z. A. Peng and X. G. Peng, J. Am. Chem. Soc. 123, 183 (2001).

${ }^{22}$ H. Zhang, Z. Zhou, B. Yang, and M. Gao, J. Phys. Chem. B 107, 8 (2003).

${ }^{23}$ W. W. Yu, L. Qu, W. Guo, and X. Peng, Chem. Mater. 15, 2854 (2003).

${ }^{24}$ See supplementary material at http://dx.doi.org/10.1063/1.3485294 for the details of the quantum dot synthesis, device processing, and reflectivity measurements. 